\title{
Erratum
}

\section{Erratum: Yao et al., "Involvement of TRPC Channels in CCL2-Mediated Neuroprotection against}

\section{Tat Toxicity"}

In the article "Involvement of TRPC Channels in CCL2-Mediated Neuroprotection against Tat Toxicity," by Honghong Yao, Fuwang Peng, Navneet Dhillon, Shannon Callen, Sirosh Bokhari, Lisa Stehno-Bittel, S. Omar Ahmad, John Q. Wang, and Shilpa Buch, which appeared on pages 1657-1669 of the February 11, 2009 issue, on page 1666, within Figure 10A, an erroneous image of the Western blot for Akt was included. This error does not affect the conclusions of the article. The authors regret the error. The updated Figure 10 is below.

A

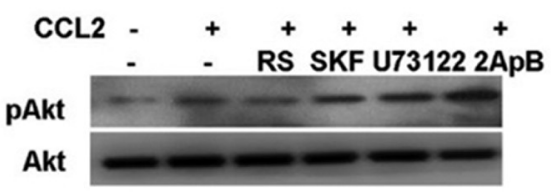

C
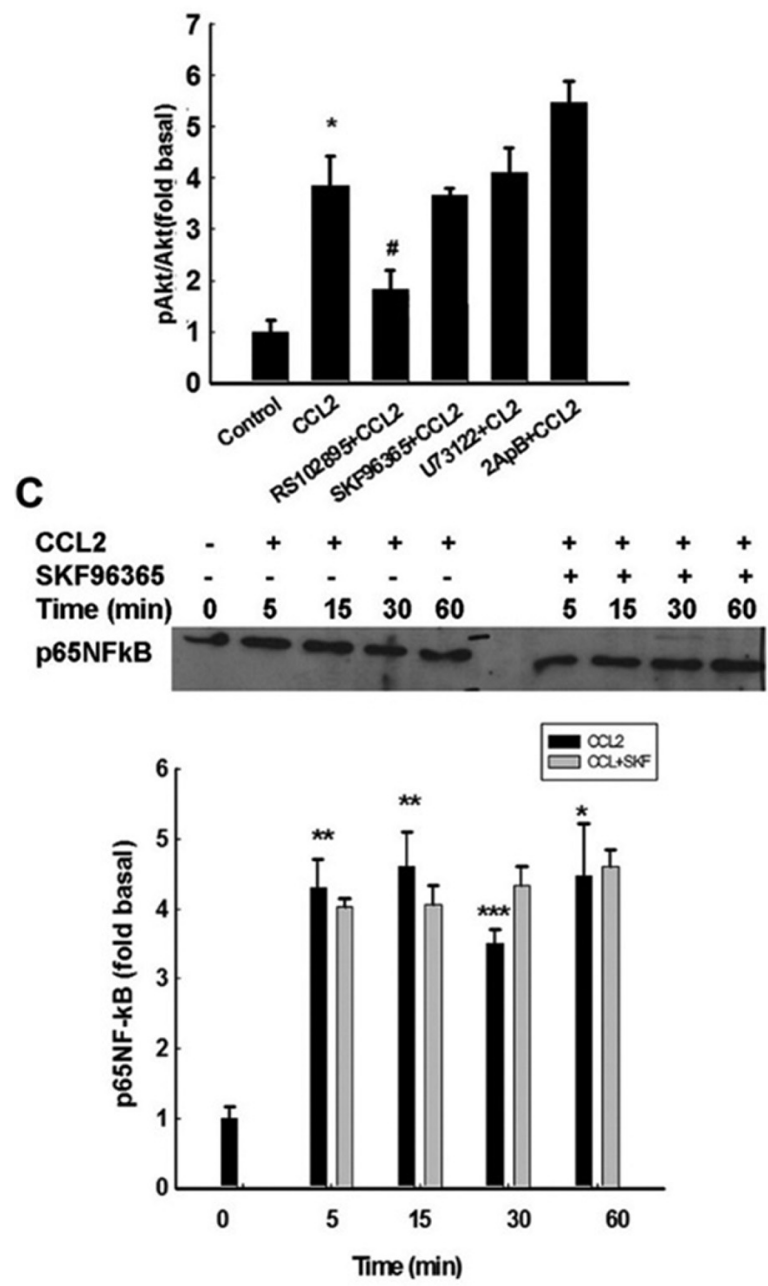

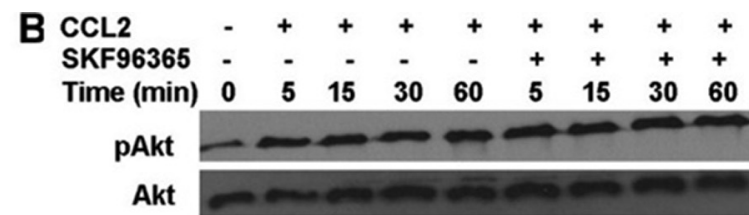

CCLL $\mathrm{CSKF}$

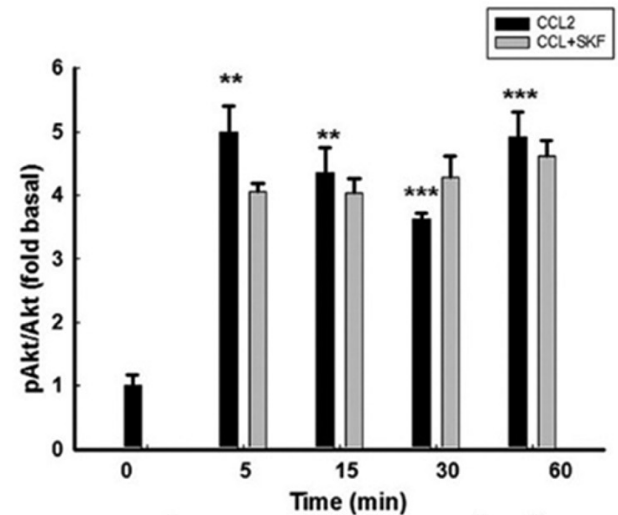

D
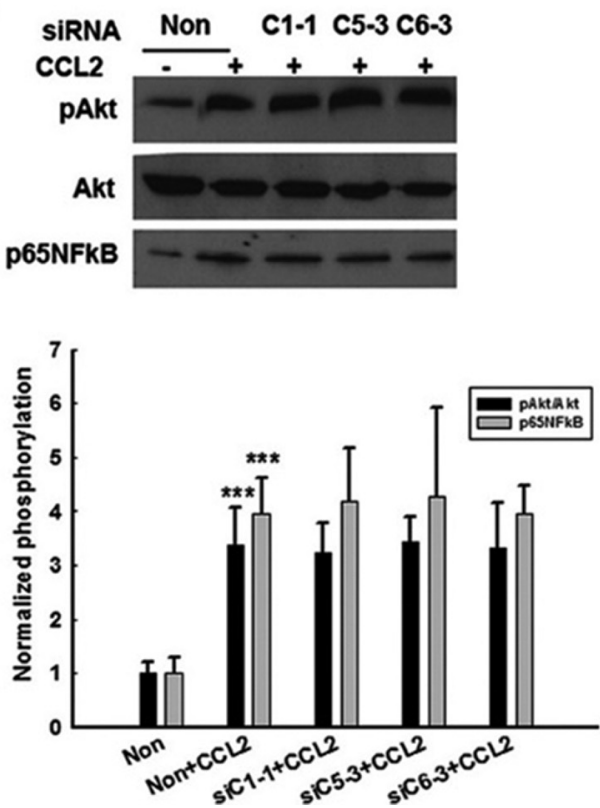

Figure 10. 